\title{
Addictive disorder
}

Robert West ${ }^{1}$

1 Univers ity College London, University of London

A chronic mental disorder realised as repeated powerful motivation to enact a behaviour, learned through experience despite experience or risk of significant harmful consequences 